\section{Reference intervals and discrimination values of the Lanthony desaturated D-15 panel test in young to middle-aged Japanese army officials: the Okubo Color Study Report 1}

T Shoji1 ${ }^{1,2,3}$, Y Sakurai ${ }^{4}$, E Chihara ${ }^{2}$, S Nishikawa ${ }^{3}$ and $\mathrm{K} \mathrm{Omae}^{1}$

\begin{abstract}
Purpose To better understand the reference values and adequate discrimination values of colour vision function with described quantitative systems for the Lanthony desaturated D-15 panel (D-15DS). Methods A total of 1042 Japanese male officials were interviewed and underwent testing using Ishihara pseudoisochromatic plates, standard pseudoisochromatic plates part 2, and the D-15DS. The FarnsworthMunsell (F-M) 100-hue test and the criteria of Verriest et al were used as definitive tests. Outcomes of the D-15DS were calculated using Bowman's Colour Confusion Index (CCI). The study design included two criteria. In criterion $A$, subjects with current or past ocular disease and a best-corrected visual acuity less than 0.7 on a decimal visual acuity chart were excluded. In criterion $B$, among subjects who satisfied criterion $A$, those who had a congenital colour sense anomaly were excluded.

Results Overall, the 90th percentile (95th percentile) CCI values for criteria $A$ and $B$ in the worse eye were 1.70 (1.95) and 1.59 (1.73), respectively. In subjects satisfying criterion $B$, the area under the receiver operating characteristic curve was 0.951 (95\% confidence interval, 0.931-0.971). The CCI discrimination values of 1.52 or 1.63 showed $90.3 \%$ sensitivity and $90 \%$ specificity, or $71.5 \%$ sensitivity and 95\% specificity, respectively, for discriminating acquired colour vision impairment (ACVI).
\end{abstract}

Conclusion We provided the 90th and 95th percentiles in a young to middle-aged healthy population. The CCI is in good agreement with the diagnosis of ACVI. Our results could be helpful for using D-15DS for screening purposes.

Eye (2009) 23, 1329-1335; doi:10.1038/eye.2008.292; published online 3 October 2008

Keywords: Lanthony desaturated D-15 panel test; Farnsworth-Munsell 100-hue test; reference interval; discrimination value; colour confusion index; colour discrimination function

\section{Introduction}

Although colour discrimination function declines with increasing age $\mathrm{e}^{1-3}$ and some occupations such as artists, graphic designers, naval navigation officers, and soldiers require good colour discrimination to perform many job-related activities, colour vision testing is generally not part of a routine screening. Little research has been performed to determine the prevalence and risk factors for acquired colour vision impairment (ACVI) in individuals of working age. The desaturated Lanthony D-15 panel (D-15DS) is most commonly used in an occupational setting ${ }^{4}$ and provides fast, accurate testing of subjects exposed to solvents ${ }^{5}$ and many chemical toxins (ie, solvent mixtures, styrene, perchloroethylene, toluene, tetrachloroethylene, carbon disulphide, and alcohol) that are lethal to the visual system. ${ }^{6-8}$
'Department of Preventive Medicine and Public Health, School of Medicine, Keio University, Tokyo, Japan

${ }^{2}$ Sensho-kai Eye Institute, Uji, Kyoto, Japan

${ }^{3}$ Department of Ophthalmology, National Defense Medical College, Tokorozawa, Saitama, Japan

${ }^{4}$ Department of Preventive Medicine and Public Health, National Defense Medical College, Tokorozawa,

Saitama, Japan

Correspondence: T Shoji, Department of Preventive Medicine and Public Health, School of Medicine, Keio University, 35, Shinanomachi, Shinjyuku-ku,

Tokyo 160-8582, Japan Tel: + 8135363 3758; Fax: +81333593686. E-mail: t-shoji@ yg7.so-net.ne.jp

Received: 2 April 2008 Accepted in revised form: 2 September 2008 Published online: 3 October 2008

The authors have no proprietary interest in any aspect of this study 
However, the test yields only semiquantitative information if analyzed by the method recommended by the manufacturer. The Farnsworth-Munsell (F-M) 100-hue test ${ }^{9}$ is time-consuming; however, it assesses acquired colour discrimination abnormalities quantitatively and is suitable for clinical and scientific research. ${ }^{1,10}$

Bowman $^{11}$ reported a method to sum up the colour differences between adjacent caps for the D-15DS calculated in the CIE L*a*b system. Bowman's Colour Confusion Index $(\mathrm{CCI})^{11}$ quantifies the total colour discrimination score as a ratio of the actual score to the ideal score.

Although some previous reports have proven that this quantitative scoring system using the D-15DS was useful for screening ${ }^{8}$ and correlated with the score of the 100-hue test, ${ }^{12,13}$ the reference intervals were investigated with only a small sample size $\mathrm{e}^{3,14,15}$ and adequate discrimination values are still unclear. In general, the 90th or 95th percentile value in a normal population is important for colour vision testing. The gold standard for the past decades for diagnosing ACVI has been the age-specific 95th percentile score of Verriest et al ${ }^{1,10,13,16}$ on the F-M 100-hue test. When the reference intervals and discrimination values of D-15DS are clearly defined, they may become more useful to screen for ACVI. The purpose of this study was to survey the distribution and adequate discrimination values of the CCI of D-15DS in a population of working-age Japanese officials.

\section{Materials and methods}

\section{Study population}

This study is a sub-study of The Okubo Color Study. The Okubo Color Study evaluates a relationship between colour vision function, especially ACVI, and ophthalmic disease, cardiovascular risk factors, and lifestyle factors. Subjects underwent annual health examinations in one community between April 2005 and March 2006 at the Okubo garrison. The authors collected physical information on current and previous diseases, use of medications, and lifestyle characteristics using structured questionnaires and personal physical records. All subjects underwent detailed interviews and measurement of visual acuity (VA), slit lamp biomicroscopy, and intraocular pressure with noncontact applanation tonometry, and also underwent a fundus examination. The VA was measured using a Landolt ring chart (with refractive correction, if any) at a distance of $5 \mathrm{~m}$. Colour fundus photographs were obtained through undilated pupils using the fundus camera system (Canon, CR-45NM, Tokyo, Japan) with an angle of $30^{\circ}$. To assess colour sense, Ishihara plates
(Kanehara Shuppan Co. Ltd, Tokyo, Japan), standard pseudoisochromatic plates part 2 (SPP2; Igaku-Shoin, Tokyo, Japan), and D-15DS plates (Luneau, Paris, France) were used. According to previous reports, ${ }^{1,15}$ colour vision tests were carried out in the morning and under daylight illumination at a luminance level of 250 lux. To maintain a luminance level, light screen or artificial lighting, entitled Standard Illuminant C, was used if necessary. All subjects underwent the same sequence of colour testing as follows: Ishihara plates, SPP2, and D-15DS. Venous blood was drawn to measure fasting plasma glucose, total cholesterol, triglycerides, lowdensity lipoprotein cholesterol, high-density lipoprotein cholesterol, uric acid, and haemoglobin A1c after an overnight fast. All examiners and photograph readers were masked to other subjects' information including vision, referring diagnosis, systemic status, and lifestyle characteristics.

All studies were reviewed by the National Defense Medical College of Research Ethics to ensure that they met the ethical guidelines, and all subjects provided written informed consent before participating according to the Helsinki Declaration 2000.

\section{Colour testing}

Ishihara pseudoisochromatic plates (Ishihara plates) Binocular inspection of Ishihara plates 1-21 (38-plate edition) was used to identify red-green colour sense abnormality. Subjects with more than four errors were classified as having failed. ${ }^{15}$

\section{Lanthony D-15DS}

The D-15DS was used to supplement standard D-15 testing to diagnose milder congenital colour anomaly or ACVI. ${ }^{5,8}$ Subjects with more than one major crossing were classified as subjects with failed results. The D-15DS was inspected monocularly, with the right eye tested before the left eye.

\section{$S P P 2$}

SPP2 was especially designed to detect ACVI, and the test also can be used to identify blue-yellow colour sense abnormalities. Subjects with more than one blue-yellow error were classified as having failed. Missing the 2 on plate 3 was not considered a failure. ${ }^{16,17}$ SPP2 was inspected monocularly, with the right eye tested before the left eye.

\section{F-M 100-hue test}

If a subject was classified as an ACVI suspect, the F-M 100-hue test was performed according to the standard technique. ${ }^{16}$ Colour discrimination was tested monocularly at a distance of $30 \mathrm{~cm}$ with the subjective 
near refraction placed in a trial frame. The subjects had abnormal hue discrimination; scores worse than the 95th percentile described by Verriest et al were diagnosed with ACVI. ${ }^{1}$ The F-M 100-hue test was tested monocularly, with the right eye tested first.

\section{Study design}

The study design is summarized in Figure 1. There were two criteria for eligibility. For criterion A, all eligible patients had to have at least a 0.7 best corrected VA (BCVA) on the decimal VA chart to see the colour plates. Subjects who had undergone cataract or retinal surgery and had a corneal or retinal disease also were excluded. To exclude side effects of drugs on colour sense, subjects who were currently taking oral digitalis glycoside, antiepileptic drugs, antimalaria drugs, or antituberculosis drugs ${ }^{16-19}$ were excluded.

For criterion B, among patients who satisfied criteria A, those who failed testing with the Ishihara plates or had a typical pattern of congenital dutan or protan defect in SPP2 or D-15DS, or a past history of congenital colour discrimination anomaly by the Ishihara plates and
D-15DS at their enlistment examination were also excluded. Questionable cases underwent anomaloscopy. Subjects who satisfied criterion B and failed the SPP2 or D-15DS in either eye were classified as ACVI suspects and were tested using the F-M 100-hue test bilaterally. Subjects exceeding the age-specific limit score of Verriest et $a l^{1}$ were diagnosed with ACVI.

\section{Outcome criteria and statistical analysis}

Data were analyzed with SPSS software, version 5.0 (SAS, Cary, NC, USA), and $P<0.05$ was considered significant. Individuals and not eyes were the unit of analysis because the right and left eyes were not examined independently. A subject was considered to have an ACVI when it occurred in either eye.

The area under the receiver operation characteristic (ROC) curve (AUC) analysis was used to assess the diagnostic values of variables for the determination of ACVI. A perfect test would have an AUC of 1, whereas a test without diagnostic value would have an AUC of 0.5. The sensitivity and specificity for detecting ACVI were determined by the F-M 100-hue test and the age-specific

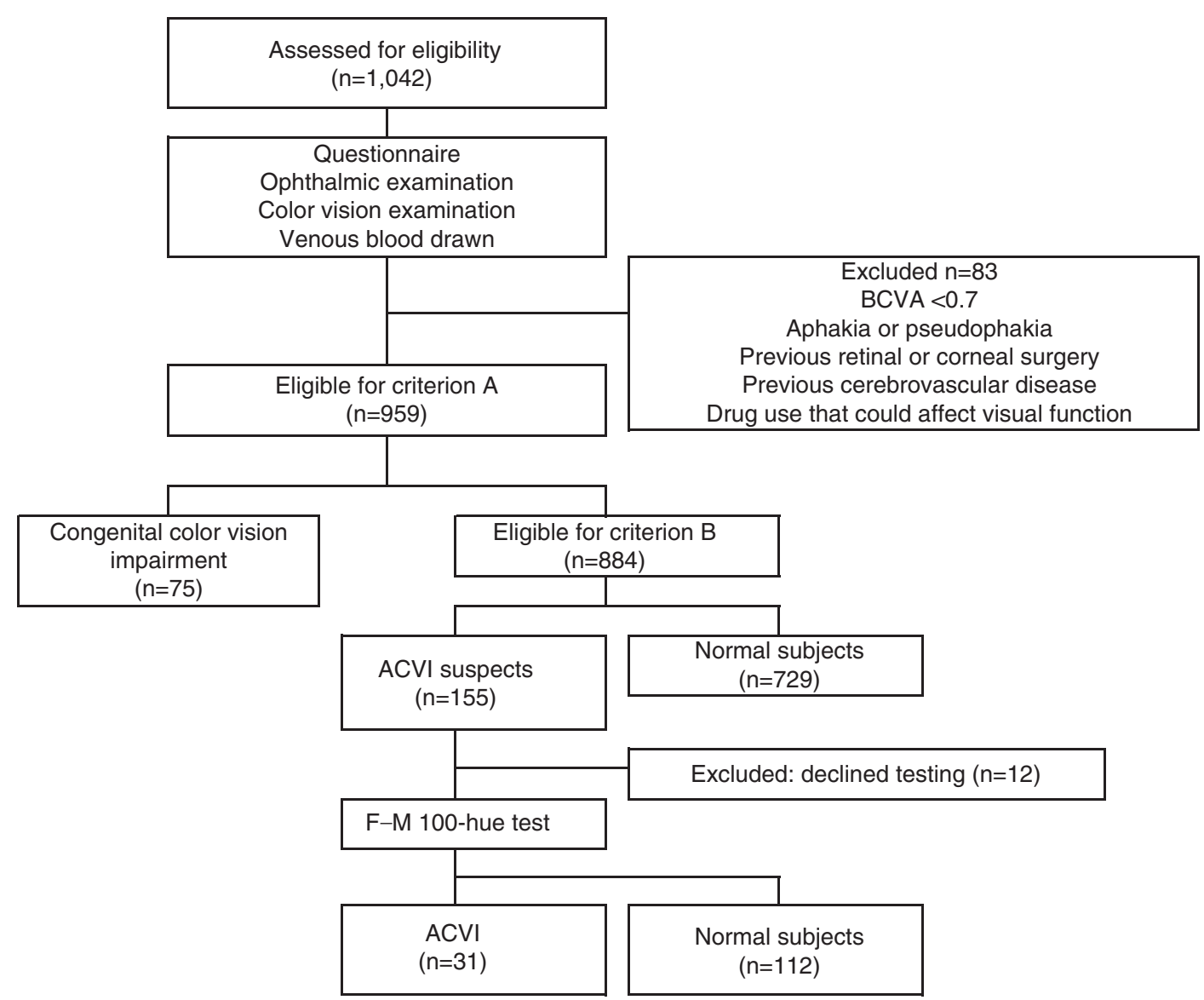

Figure 1 Schematic diagram of The Okubo Color Study. Diagnostic schema of ACVI. 
criteria of Verriest et al. ${ }^{1}$ This analysis was applied to eligible subjects who satisfied criterion B; subjects who declined to undergo the F-M 100-hue test were excluded.

According to the guidelines, ${ }^{20}$ the serum low-density lipoprotein cholesterol level was categorized as less than $130 \mathrm{mg} / 100 \mathrm{ml}$, equal to or exceeding $130 \mathrm{mg} / 100 \mathrm{ml}$ but less than $160 \mathrm{mg} / 100 \mathrm{ml}$, or equal to or exceeding $160 \mathrm{mg} / 100 \mathrm{ml}$. Systemic hypertension was defined as a systolic/diastolic pressure of $140 / 90 \mathrm{mmHg}$ or more at the time of examination, or a history of diagnosis of hypertension and current use of medication for hypertension. ${ }^{20}$ Diabetes was defined as a previous history of diabetes treated with either insulin, oral hypoglycaemia agents, or diet. Newly diagnosed diabetes was defined as no previous medical history of diabetes in the presence of elevated glycated haemoglobin or a fasting blood glucose level of $126 \mathrm{mg} / 100 \mathrm{ml}$ or more at the time of examination. ${ }^{21}$ Hyperuricemia was defined as a serum uric acid value of $8.0 \mathrm{mg} / 100 \mathrm{ml}$ or more at the time of examination, or a history of gout and current use of medication for hyperuricemia.

\section{Smoking status}

Subjects were categorized as those who have never smoked, current, and prior smokers. Current and prior smokers were defined as current or past habitual smokers who had smoked for at least 1 year. Subjects who had been smoking for less than 1 year were not considered to be smokers. Cigarette smokers were classified into light and heavy smokers based on the cigarette pack-years smoked, which indicates the personal amount of smoking over a long period. Cigarette pack-years were calculated by multiplying the number of packs of cigarettes smoked per day by the number of years the person had smoked. For example, 1 pack-year is equal to smoking one pack per day for 1 year. In this analysis, subjects were considered to be light smokers if they had fewer than 19 pack-years of smoking (median pack-years of smoking) and heavy smokers if they had 19 or more pack-years of smoking. We checked the medical records and the subjects' response to questionnaires to confirm past and current diseases.

\section{Results}

The demographic data are shown in Table 1. A total of 959 and 884 subjects, respectively, were eligible for criteria A and B. Table 2 shows the number of subjects and the mean/median CCIs in each age group. The median CCIs in each age group were similar between criteria A and B. Figure 2 shows the distribution of CCI in
Table 1 Baseline characteristics of eligible subjects

\begin{tabular}{lcc}
\hline & Criterion A & Criterion B \\
\hline Number of subjects & 959 & 884 \\
Age (years) & $38.0 \pm 8.7$ & $37.8 \pm 8.7$ \\
Height $(\mathrm{cm})$ & $170.2 \pm 5.9$ & $170.2 \pm 5.9$ \\
Weight $(\mathrm{kg})$ & $68.5 \pm 9.1$ & $68.6 \pm 9.0$ \\
Body mass index $\left(\mathrm{kg} / \mathrm{m}^{2}\right)$ & $23.7 \pm 2.7$ & $23.6 \pm 2.7$ \\
& & \\
& Number of & Number of \\
& subjects (\%) & subjects (\%) \\
\hline Hypertension & $121(12.6)$ & $110(12.4)$ \\
& & \\
LDL cholesterol, $m g / 100 \mathrm{ml}$ & & \\
$\quad<130$ & $614(64.0)$ & $561(63.5)$ \\
130-159 & $231(24.1)$ & $215(24.3)$ \\
$\quad 160$ & $114(11.9)$ & $108(12.2)$ \\
Hyperuricemia & $195(20.3)$ & $187(21.2)$ \\
Diabetes & $20(2.1)$ & $17(1.9)$ \\
& & \\
Smokers & & $340(38.5)$ \\
$\quad$ Never smoked & $374(39.0)$ & $274(31.0)$ \\
Light smoker & $293(30.6)$ & $270(30.5)$ \\
Heavy smoker & $292(30.4)$ & \\
\hline
\end{tabular}

\pm values are means $\pm \mathrm{SD}$

Table 2 Numbers of subjects and, median and mean CCI for each criterion by age group

\begin{tabular}{|c|c|c|c|c|c|c|}
\hline \multirow{2}{*}{$\begin{array}{l}\text { Age } \\
\text { (years) }\end{array}$} & \multicolumn{3}{|c|}{ Criterion A } & \multicolumn{3}{|c|}{ Criterion B } \\
\hline & No. & Median & $\begin{array}{c}\text { Mean } \\
(95 \% \text { CI) }\end{array}$ & No. & Median & $\begin{array}{c}\text { Mean } \\
(95 \% \text { CI })\end{array}$ \\
\hline $20-29$ & 228 & 1.22 & $1.28(1.25-1.31)$ & 209 & 1.21 & $1.26(1.23-1.29)$ \\
\hline $30-39$ & 225 & 1.19 & $1.27(1.23-1.31)$ & 210 & 1.18 & $1.23(1.20-1.26)$ \\
\hline $40-49$ & 451 & 1.28 & $1.38(1.34-1.42)$ & 416 & 1.24 & $1.29(1.27-1.31)$ \\
\hline $50-59$ & 55 & 1.28 & $1.38(1.26-1.50)$ & 49 & 1.20 & $1.28(1.20-1.36)$ \\
\hline Total & 959 & 1.22 & $1.33(1.31-1.35)$ & 884 & 1.22 & $1.27(1.25-1.30)$ \\
\hline
\end{tabular}

the worse eye, with worse defined as the personal worse score between the right and left eyes of one subject. The $\mathrm{CCI}$ in the worse eye did not have a normal distribution even after transformation $(P<0.001$ KolmogorovSmirnov test).

Figure 3 shows the age-specific 90th and 95th percentile CCIs that met criteria $\mathrm{A}$ and $\mathrm{B}$, respectively. In all subjects, the 90 th percentile (95th percentile) scores in the worse eye were 1.70 (1.95) in criteria A and 1.59 (1.73) in criteria B, respectively. Figure 4 shows the ROC curve of the worse CCI values. AUC was 0.951 (95\% confidence interval (CI), 0.931-0.971). For this algorithm, specificities of $80,85,90$, and $95 \%$ were reached for sensitivities of $96.8,93.6,90.3$, and $71.0 \%$, respectively (Table 3 ). 


\section{Discussion}

This study determined the age-specific normal range of $\mathrm{CCI}$ in working-age Japanese men and indicated that the $\mathrm{CCI}$ is in good agreement with the diagnosis of ACVI using the F-M 100-hue test.

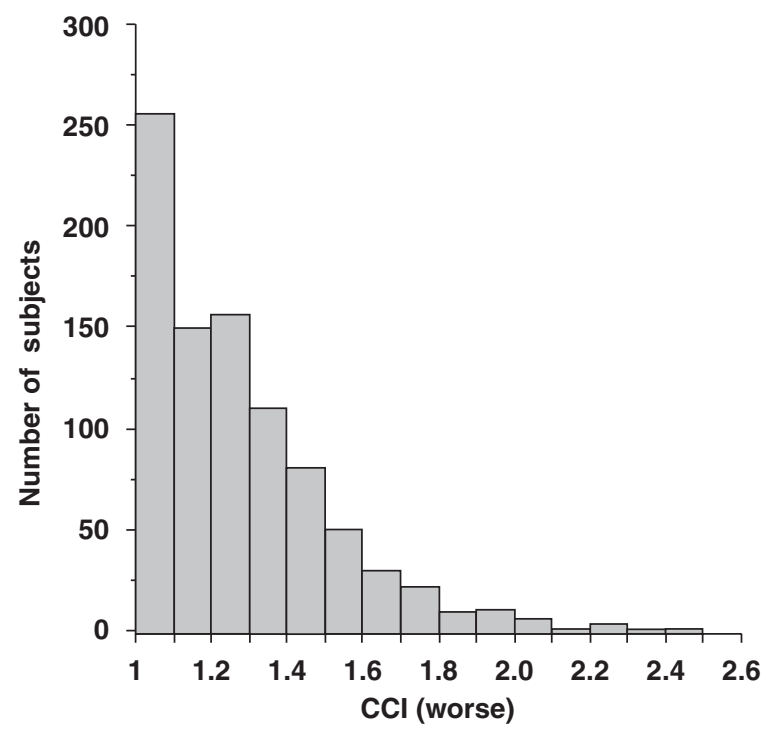

Figure 2 Distribution of CCI in the worse eye (eligible for criterion B).

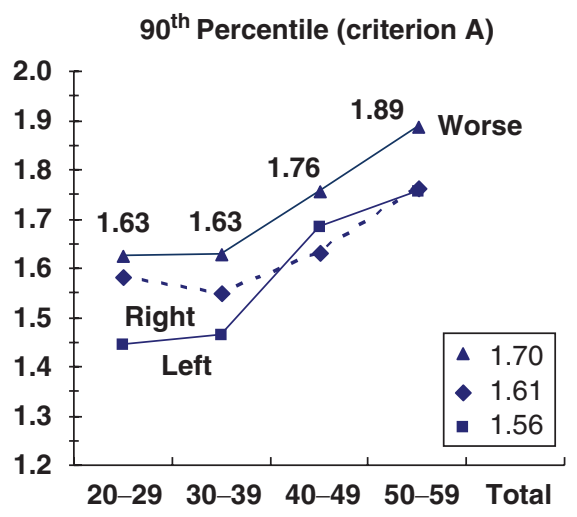

95 ${ }^{\text {th }}$ Percentile (criterion A)

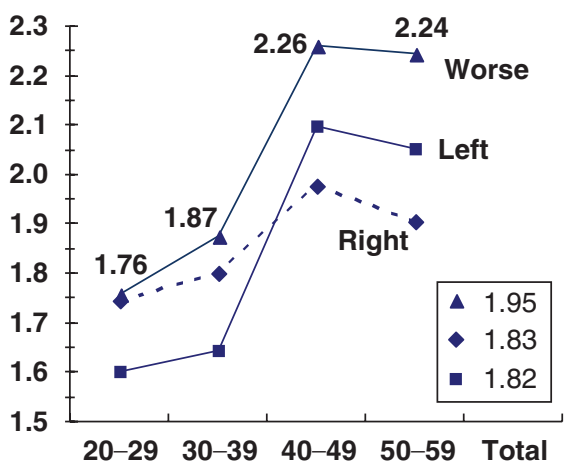

Surveillance of reference intervals and discrimination values is important because previous reports have described that ACVI can precede deterioration of VA or be correlated with an ocular disease such as cataract, ${ }^{22}$ diabetic retinopathy, ${ }^{10}$ glaucoma, age-related macular degeneration, and lifestyle factors such as acute and

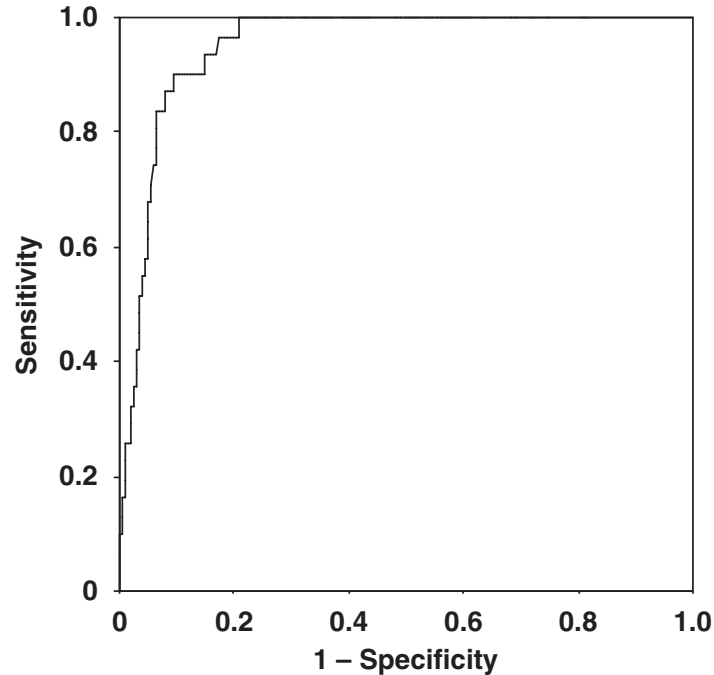

Figure 4 ROC curve analysis. The AUC is 0.951 (95\% CI, 0.931-0.971).
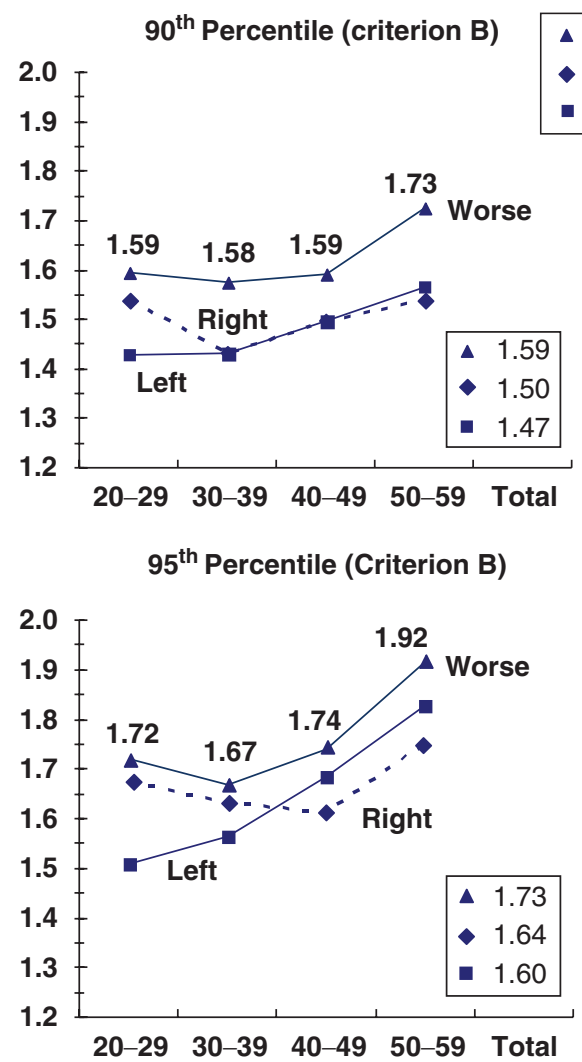

Figure 3 The 90th and 95th percentile limits of CCI by age. 
Table 3 Sensitivity, decision threshold value of CCI, and positive predictive rate at a fixed specificity

\begin{tabular}{lccc}
\hline $\begin{array}{l}\text { Decision threshold } \\
\text { value of CCI }\end{array}$ & $\begin{array}{c}\text { Sensitivity } \\
(\%)\end{array}$ & $\begin{array}{c}\text { Positive predictive } \\
\text { rate }(\%)\end{array}$ & $\begin{array}{c}\text { Specificity } \\
(\%)\end{array}$ \\
\hline 1.40 & 96.8 & 15.2 & 80 \\
1.44 & 93.6 & 18.8 & 85 \\
1.52 & 90.3 & 25.7 & 90 \\
1.63 & 71.0 & 30.0 & 95 \\
\hline
\end{tabular}

chronic ethanol intake, and tobacco smoking. ${ }^{4,6,8,23,24}$ Moreover, extended exposure to numerous chemical agents and medicine also has been associated with alterations in colour discrimination. ${ }^{6-8,19}$ Although those case-control studies were well reported, multifactorial analysis of ACVI has been studied less. The Okubo Color Study will investigate these relationships in later reports.

Colour vision testing generally provides a wide range of reference intervals in clinical work. ${ }^{16}$ In previous reports by Ihrig et $a l_{,}^{25}$ the median CCI value was 1.00-1.17 in 238 normal German subjects (mean age, 27 years). Good et $a l^{14}$ reported that 54 normal men without ocular or systemic disease scored 1.10 (average) on the primary D-15DS test in 1400 lux illumination. Iregren $e t$ al $^{15}$ reported a median CCI score of 1.13 in 199 healthy volunteers or 1.16 in 1000 lux illumination. Urban et $a l^{7}$ reported that 24 healthy subjects (mean age, 42 years) scored 1.04. These values were better than the values obtained in this study. The reason for these discrepancies is unknown, but several factors can be considered. First, Woo ${ }^{26}$ reported that differences in macular pigmentation between Caucasians and Asians cause substantial differences in the results of the F-M 100-hue test. Our entire study population was Japanese, which might have contributed to the difference. Second, we analyzed the worse eye score, and individuals rather than eyes were the unit of analysis because the right and left eyes were not independent. Our methodology might have contributed to the discrepancy. Third, standardized conditions, especially the illumination level, might be important when administering the D-15DS. The differences in light luminance may partly explain the differences.

To the best of our knowledge, few studies have been published on reference intervals and discrimination CCI scores. Regarding the 90th percentile score, Iregren et $\mathrm{ll}^{15}$ tested 199 subjects, after excluding congenital colour vision deficits, and reported that the overall 90th percentile CCI scores were 1.54 in right eyes and 1.56 in left eyes. In this study, the overall 90th percentile score in the subjects who met criteria B was 1.50 in the right eyes and 1.47 in the left eyes (Figure 3 ), which are close to the results reported by Iregren et al. ${ }^{15}$ Regarding the 95th percentile score, Ihrig et $a l^{25}$ tested patients binocularly and reported that the CCI values in a healthy group ranged from 1.21 to 1.61 calculated by the mean and SD. Unfortunately, unlike the F-M 100-hue test scores, the 90th and the 95th percentile CCI scores cannot be calculated using the mean and SD, because the CCI using D-15DS does not have a Gaussian distribution even after transformation $^{3}$ (Figure 2). Therefore, a large sample size is required to assume the accuracy of the 90th or 95th percentile score. Significantly better results with the patients who satisfied criterion B compared with those who satisfied criterion A implied that a small number of subjects with impaired congenital colour discrimination strongly affect the limit value rather than the median value (Table 2).

Bassi et $a l^{13}$ reported that in 35 patients with glaucoma included in an ACVI model, there is a significant correlation between the total error scores in the F-M 100hue test and the C-index, which is another quantitative system, in the D-15DS. However, the investigators excluded participants with normal colour function and did not describe the discrimination value. In this study, when the discrimination value of the CCI was set to 1.52 , there was good agreement with the diagnosis of ACVI using the longer F-M 100-hue test in an epidemiologic study (sensitivity, 90.2\%; specificity, 90\%). This score may be the best discrimination value.

In the current results, the reference value of $\mathrm{CCI}$ in the right eye was significantly higher than that in the left eye in only the youngest age group (Figure 3 ). The reason is unclear, but our methodology, in which the right eye was tested before the left eye, might have affected the results. In general, colour vision testing is affected by the learning curve or fatigue but it has been studied less. We plan to investigate these effects in later studies.

Nevertheless, the differences affected our results less, because individuals were the unit of analysis not eyes.

A limitation of this study was that the subjects were all male Japanese officials on active duty. Research among women, individuals in other occupations, or individuals of other races may be necessary.

In addition, the illumination level might be important when administering the D-15DS. Iregren et $a l^{6}$ and Gobba $^{8}$ reported that they applied the D-15DS in the morning using daylight fluorescent lamp providing 1200 lux. Baird et $a l^{27}$ reported that the D-15DS required a 500-lux Macbeth lamp light. Melamud reported that 100 lux should be the minimal level of illumination for screening. ${ }^{16}$ However, we do not know whether or how the level of illumination used during testing might have affected the test results. ${ }^{27}$ In our results, the median CCI was higher than the results reported by Iregren, but the 90th percentile CCI was similar. ${ }^{15}$ Thus, the difference in 
the illumination level may be less important when discriminating between ACVI and healthy subjects.

Another limitation was related to positive prevalence rate, which is the chance of having a disease if one screens positive, and it is prevalence dependent. Even with high sensitivity and specificity values, the positive predictive rate is low if the prevalence is low. Because of the low prevalence of ACVI in this study, the CCI with the D-15DS also shows a low positive predictive rate (Table 3). Thus, the F-M 100-hue test cannot be replaced by the D-15DS completely and will remain the definitive test.

In conclusion, our study provided the normal healthy distribution in a large number of working-aged men on active duty using the D-15DS test with the CCI scoring system. Our results could be helpful for clinicians and patients when the D-15DS test is performed for screening purposes.

\section{Acknowledgements}

The authors thank The Okubo Color Study team, in particular, K Tanaka for subject recruitment; A Katagiri for helping in preparing the study; K Sintomi, Y Usuda, T Kanazaki, N Nakai, Y Komatsu, and T Bessho for clinical input; and the volunteers for participating in the study. In addition, we thank Ms Lynda Charters for her linguistic and editorial support.

\section{References}

1 Verriest G, Van Laethem J, Uvijls A. A new assessment of the normal ranges of the Farnsworth-Munsell 100-hue test scores. Am J Ophthalmol 1982; 93: 635-642.

2 Mantyjarvi M. Normal test scores in the FarnsworthMunsell 100-hue test. Doc Ophthalmol 2001; 102: 73-80.

3 Roy MS, Podgor MJ, Collier B, Gunkel RD. Color vision and age in a normal North American population. Graefes Arch Clin Exp Ophthalmol 1991; 229: 139-144.

4 Gobba F, Cavalleri A. Color vision impairment in workers exposed to neurotoxic chemicals. Neurotoxicology 2003; 24: 693-702.

5 Gobba F, Cavalleri A. Evolution of color vision loss induced by occupational exposure to chemicals. Neurotoxicology 2000; 21: 777-781.

6 Iregren A, Andersson M, Nylen P. Color vision and occupational chemical exposures: I. An overview of tests and effects. Neurotoxicology 2002; 23: 719-733. Review.

7 Urban P, Gobba F, Nerudova J, Lukas E, Cabelkova Z, Cikrt M. Color discrimination impairment in workers exposed to mercury vapor. Neurotoxicology 2003; 24: 711-716.

8 Gobba F. Related Color vision: a sensitive indicator of exposure to neurotoxins. Neurotoxicology 2000; 21: 857-862.
9 Farnsworth D. The Farnsworth-Munsell 100-Hue Test Manual (Revised). Munsell Color Co.: Baltimore, 1957.

10 Fong DS, Barton FB, Bresnick GH. Impaired color vision associated with diabetic retinopathy: Early Treatment Diabetic Retinopathy Study Report No. 15. Am J Ophthalmol 1999; 128: 612-617.

11 Bowman KJ. A method for quantitative scoring of the Farnsworth Panel D-15. Acta Ophthalmol (Copenh) 1982; 60: 907-916.

12 Vingrys AJ, King-Smith PE. A quantitative scoring technique for panel tests of color vision. Invest Ophthalmol Vis Sci 1988; 29: 50-63.

13 Bassi CJ, Galanis JC, Hoffman J. Comparison of the Farnsworth-Munsell 100-Hue, the Farnsworth D-15, and the L'Anthony D-15 desaturated color tests. Arch Ophthalmol 1993; 111: 639-641.

14 Good GW, Schepler A, Nichols JJ. The reliability of the lanthony desaturated D-15 test. Optom Vis Sci 2005; 82: 1054-1059.

15 Iregren A, Andersson M, Nylen P. Color vision and occupational chemical exposures. II. Visual functions in non-exposed subjects. Neurotoxicology 2002; 23: 735-745.

16 Melamud A, Hagstrom S, Traboulsi E. Color vision testing. Ophthalmic Genet 2004; 25: 159-187.

$17 \mathrm{Vu}$ BL, Easterbrook M, Hovis JK. Detection of color vision defects in chloroquine retinopathy. Ophthalmology 1999; 106: 1799-1803.

18 Gobba F, Cavalleri A. Color vision impairment in workers exposed to neurotoxic chemicals. Neurotoxicology 2003; 24: 693-702.

19 Lawrenson JG, Kelly C, Lawrenson AL, Birch J. Acquired colour vision deficiency in patients receiving digoxin maintenance therapy. Br J Ophthalmol 2002; 86: 1259-1261.

20 Third Report of the National Cholesterol Education Panel (NCEP) Expert Panel on Detection, Evaluation, and Treatment of High Blood Cholesterol in Adults (Adult Treatment Panel or ATP III). NIH Publication No. 01-3305 May 2001.

21 American Diabetes Association. Standards of medical care in diabetes-2006. Diabetes Care 2006; 29(Suppl 1): S4-S42.

22 Kessel L, Alsing A, Larsen M. Diabetic versus non-diabetic colour vision after cataract surgery. Br J Ophthalmol 1999; 83: 1042-1045.

23 Cavalleri A, Gobba F, Nicali E, Fiocchi V. Dose-related color vision impairment in toluene-exposed workers. Arch Environ Health 2000; 55: 399-404.

24 Alcala A, Morell M, Rius F. Comparative study of the impact of diet versus pravastatin on color vision in Brodman area 19 detected by computerized chromatic analysis (CARDIOCOLOUR Study). Rev Esp Cardiol 2002; 55: 1243-1250.

25 Ihrig A, Nasterlack M, Dietz MC, Hoffmann J, Triebig G. Pilot study on prevalence of color vision dysfunction in long-term solvent-exposed painters. Ind Health 2003; 41: 39-42.

26 Woo GC. Are ethnic differences in the F-M 100 scores related to macular pigmentation? Clin Exp Optom 2002; 85: 372-377.

27 Baird B, Camp J, Daniell W, Antonelli J. Solvents and color discrimination ability. Nonreplication of previous findings. J Occup Med 1994; 36: 747-751. 